\title{
Appearance of a Robot Influences Causal Relationship between Touch Sensation and the Personality Impression
}

\author{
Yuki Yamashita \\ Osaka University \\ Osaka, Japan \\ yuki.yamashita@ \\ ams.eng.osaka-u.ac.jp
}

\author{
Hisashi Ishihara \\ Osaka University \\ Osaka, Japan \\ ishihara@ \\ ams.eng.osaka-u.ac.jp
}

\author{
Takashi Ikeda \\ Kanazawa University \\ Kanazawa, Japan \\ tikeda@ \\ med.kanazawa-u.ac.jp
}

\author{
Minoru Asada \\ Osaka University \\ Osaka, Japan \\ asada@ \\ ams.eng.osaka-u.ac.jp
}

\begin{abstract}
Personality impressions of robots have been regarded as one of the crucial factors in human-robot interaction. To design the personality impressions, we should know how the visual, auditory, and tactile impressions determine the personality impressions. In this study, we investigated the relationships between touch sensations and personality impressions with a child-type android robot in two conditions where 40 Japanese participants touched a part of the robot with different appearance of the face. Factor and path analyses were conducted on the evaluation scores of the sensations and impressions provided by the participants. As a result, two significant positive causal relationships $(\mathrm{p}<0.001)$ were found between the Preference and Resilience touch sensations, and the Likability and Capability personality impressions, respectively, in both robot conditions. On the other hand, several other causal relationships were found only in one condition. This suggests that there are appearance-dependent and appearance-independent relationships between touch sensations and personality impressions.
\end{abstract}

\section{Author Keywords}

Touch sensation; Personality impression; Factor analysis; Path analysis; Android robot; Human-robot interaction; Social robotics

\section{ACM Classification Keywords}

\section{SOCIAL AND BEHAVIORAL SCIENCES}

\section{INTRODUCTION}

Improving a human's impression of robots is an important but difficult challenge in human-robot interaction $[11,13,20]$. One of the reasons for the difficulty in impression design is that we know empirically but not systematically how each of the several features of a robot changes the human's impression of the robot as a whole. To overcome this issue, a number of studies examined the causal relationships

Permission to make digital or hard copies of part or all of this work for personal or classroom use is granted without fee provided that copies are not made or distributed for profit or commercial advantage and that copies bear this notice and the full citation on the first page. Copyrights for thirdparty components of this work must be honored. For all other uses, contact the owner/author(s). Copyright is held by the author/owner(s).

$H A I^{\prime} 17$, October17-20, 2017, Bielefeld, Germany

(C) 2017 Copyright is held by the owner/author(s).

ACM ISBN 978-1-4503-5113-3/17/10.

https://doi.org/10.1145/3125739.3132587 between robot features, such as its appearance and voice, and the personality impressions of the robot $[1,2,10,15,16,17,19]$. In contrast, touch sensations and overall impressions (or personality impressions) of robots have only been investigated independently $[4,16,22]$ in most cases. The relationships between them should be investigated because the opportunities for humans to touch robots are expected to increase, and the humans' impressions are important for communication robots.

We analyzed the relationships between touch sensations and personality impressions of a robot [24], which was a child android robot called "Affetto" [7], by using a path and factor analyses method. They found several causal relationships between four components of touch sensations, i.e., Preference, Resilience, Smoothness, and Naturalness, and three components of personality impressions, i.e., Likability, Capability, and Vitality [24]. These components were found by a factor analysis method for evaluating scores of the touch sensations and personality impressions of the robot provided by Japanese adult participants. The found causal relationships help robot designers to decide touch sensations of their robots that give the intended personality impressions.

However, it is unknown whether the causal relationships found in the previous study are robust enough not to be eliminated or the other causal relationships are enhanced if the appearance of the robot is different from that of the one in the previous study. Therefore, in this study, we compared the differences in the causal relationships between two conditions: the Android condition, in which the face skin was set to Affetto, and the Humanoid condition, in which face skin was removed from Affetto's face.

\section{METHOD}

\section{Participants}

Forty healthy adults participated in this experiment. Twenty participants (10 females and 10 males, mean age $=21.8, \mathrm{SD}$ $=1.7$ ) were assigned to the Android condition, and twenty participants (10 females and 10 males, mean age $=22.4$, SD $=1.9$ ) were assigned to the Humanoid condition.

Robot

Figure 1 shows the appearances of the child android robot "Affetto" we used in this study in the two experimental conditions. For the Android condition, we used Affetto with 
its facial skin so that its face was covered with a human-like silicone rubber skin, as shown in Fig. 1 (a). On the other hand, for Humanoid condition, we used Affetto without its facial skin so that the internal mechanisms of its face were exposed, as shown in Fig. 1 (b). This condition setting enabled us to highlight the visual difference without changing the condition of the robot forearm which were touched by the participants. In both conditions, Affetto was set on a desk in front of a chair, on which the participants sat. Almost all the parts of the robot were covered by clothes and gloves, whereas its face and left forearm were exposed. All the joints of the robot were fixed so that its posture could be maintained when the participants touched it. Its left forearm could be replaced with each of four forearms A, B, C, and D with different surface hardness, and identical sizes and appearances. These forearms are made by silicone rubber (Dragon Skin Fx-Pro, Smooth-On Inc.) and their surface were wrapped with a thin polyurethane film (Airwall UV, Kyowa Ltd.). The hardness was measured by a durometer (ASKER Durometer Type FP, Kobunshi Keiki Co., Ltd.) and forearm $\mathrm{A}$ is harder than co-constructed upper arm of adult males, B, C and D is similar to forceful upper arm of adult males, the relaxed forearms of males (or the forceful upper arm of females) and the relaxed upper arm of females.

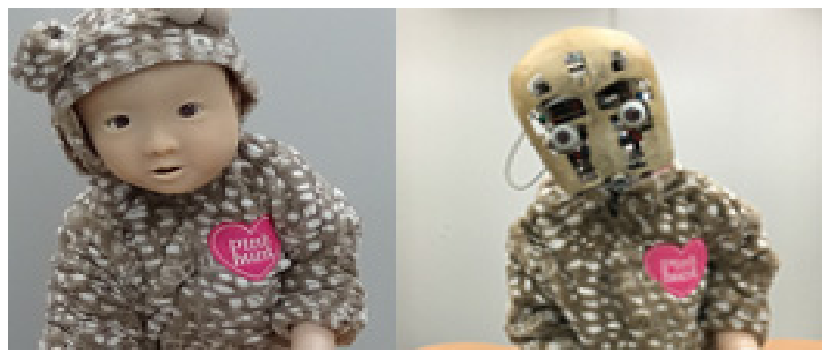

(a)

(b)

Figure 1. Appearance of the child android robot "Affetto" in the two experimental conditions: Android condition (a) and Humanoid condition (b).

\section{Questionnaire}

A seven-point semantic differential method (SD method) [18] was used to evaluate touch sensations and personality impressions. Two sets of questionnaires to evaluate touch sensations and personality impressions were provided to the participants. These questionnaires were composed of 19 Japanese adjective pairs for touch sensation, e.g., "Soft or Hard" and "Comfortable or Uncomfortable," and 46 pairs for personality impression, e.g., "Talkative or Reticent" and "Reliable or Unreliable." These adjective pairs were introduced in the study done by Yamashita et al. [24]. This list was created by merging several lists of adjective pairs related to touch sensations and personality impression in several studies $[3,5,6,8,12,16,22]$ to collect participants' evaluations exhaustively.

\section{Procedure}

At first, the participants watched instruction movie explaining the manner of touching robot's forearm. The participants were instructed to touch and grab the exposed part of the left forearms of Affetto with their dominant hand, as shown in Figure 2. Then, they were instructed to answer the questionnaires for touch sensations. They were then instructed to touch and grab Affetto's forearm again, and then instructed to answer the questionnaires for personality impressions. The above procedures were repeated four times with each of four forearms A, B, C, and D in a randomized order.

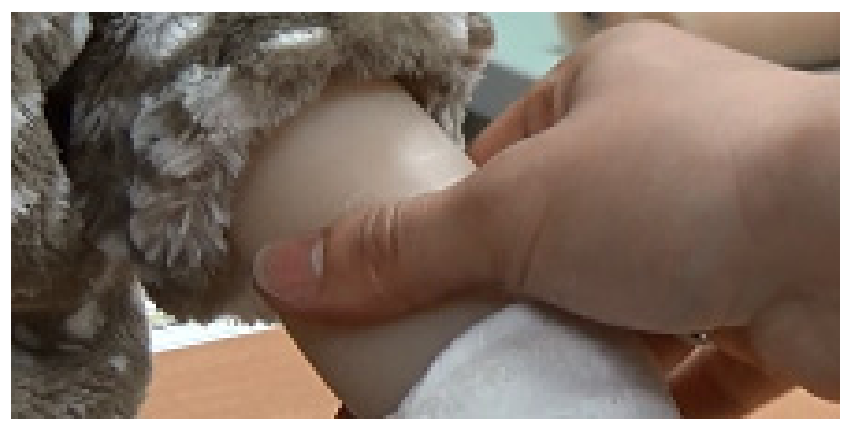

Figure 2. Actual experimental situation where a participant has grabbed the exposed part of the left forearm of Affetto.

\section{Data processing}

We conducted an exploratory factor analysis using the maximum-likelihood with promax rotation to identify underlying factors, each of which was statistically reflected by several evaluation scores of the adjective pairs. The number of factors was determined based on the scree test and was investigated by the Bayesian information criterion (BIC) and the root mean square error of approximation (RMSEA). We conducted path analyses to find significant causal relationships between the found factors in both conditions. We employed the multivariate multiple regression model whose independent and dependent variables were the touch sensation factors and the personality impression factors. Multicollinearity among variables was diagnosed with the variance inflation factor (VIF). The model parameters were estimated by the maximum-likelihood estimation. For the above analyses, we used the R language version 3.2.2 [23].

\section{RESULT}

\section{Factor analysis}

Factor analyses of each questionnaire extracted four and three factors in touch sensations and personality impressions, respectively. These numbers of factors were determined by inspecting the scree plot of eigenvalues. BIC and RMSEA were -350.78 and 0.067 for the touch sensation factors, while they were -3016.45 and 0.077 for the personality impression factors.

For touch sensation, we named factor 1 as "Preference," factor 2 as "Resilience," factor 3 as "Smoothness," and factor 4 as "Roundness," while for personality impression, we named factor 1 as "Likability," factor 2 as "Capability," and factor 3 as "Vitality." (Table 1 and 2). 


\begin{tabular}{|c|c|c|c|c|}
\hline & \multicolumn{4}{|c|}{ Factor } \\
\hline & 1 & 2 & 3 & 4 \\
\hline & $\begin{array}{l}\text { Prefer- } \\
\text { ence }\end{array}$ & $\begin{array}{l}\text { Resili- } \\
\text { ence }\end{array}$ & $\begin{array}{c}\text { Smooth- } \\
\text { ness }\end{array}$ & $\begin{array}{c}\text { Round- } \\
\text { ness }\end{array}$ \\
\hline Desirable & .93 & & & \\
\hline Pleasant & .93 & & & \\
\hline Good-feeling & .92 & & & \\
\hline Good & .86 & & & \\
\hline Tense & & .91 & & \\
\hline Supple & & .90 & & \\
\hline Rigid & & .76 & & \\
\hline Hard & & .68 & & \\
\hline Smooth & & & .78 & \\
\hline Fine & & & .53 & \\
\hline Rounded & & & & .65 \\
\hline
\end{tabular}

Table 1. Factor matrix for touch sensation factors; loadings higher than an absolute value of 0.50 are shown.

\begin{tabular}{|c|c|c|c|}
\hline & \multicolumn{3}{|c|}{ Factor } \\
\hline & 1 & 2 & 3 \\
\hline & Likability & Capability & Vitality \\
\hline Agreeable & .93 & & \\
\hline Comfortable & .92 & & \\
\hline Pleasant & .89 & & \\
\hline Desirable & .87 & & \\
\hline Good & .84 & & \\
\hline Soothing & .84 & & \\
\hline Adorable & .83 & & \\
\hline Friendly & .81 & & \\
\hline Reassuring & .79 & & \\
\hline Approachable & .78 & & \\
\hline Merry & .75 & & \\
\hline Amiable & .74 & & \\
\hline Robust & & .86 & \\
\hline Reliable & & .85 & \\
\hline Strong & & .85 & \\
\hline Brave & & .77 & \\
\hline Confident & & .77 & \\
\hline Talkative & & & .79 \\
\hline Noisy & & & .75 \\
\hline
\end{tabular}

Table 2. Factor matrix for personality impression factors; loadings higher than an absolute value of 0.70 are shown.

\section{Path analysis}

The factor scores calculated from the factor analyses were used for path analyses to investigate how each of the touch sensation factors affected each of the personality impression factors in each condition. Satisfactory goodness-of-fit index (RMSEA < 0.001) for a full model, including all of the possible paths, was achieved.

Figures 3 and 4 show the path diagrams in the Android and Humanoid conditions, respectively. In each diagram, path coefficients between touch sensation factors and personality impression factors are represented. The insignificant paths $(p$ $>0.05$ ) were removed. The thickness of each path represents the magnitude of the coefficient. Solid and dotted lines represent positive and negative causal relationships, respectively. Multicollinearity between each touch sensation factor and personality impression factor was not observed (all VIF values were less than 5 ).

In the Android condition, we found that the Likability personality impression was highly improved by the Preference touch sensation $(\beta=.918)$ and slightly improved by the Resilience and Roundness touch sensations $(\beta=.092$ and .181 , respectively); the Capability personality impression was improved by the Resilience and Preference touch sensations $(\beta=.773$ and .172 , respectively), but worsened by the roundness touch sensation $(\beta=.388)$; and the Vitality personality impression was improved by the Preference, Roundness, and Resilience touch sensations ( $\beta$ $=.349, .365$, and .314 , respectively).

On the other hand, in the Humanoid condition, we found only two significant causal relationships: The Likability personality impression was highly improved by the Preference touch sensation $(\beta=.763)$, and the Capability personality impression was improved by the Resilience touch sensation $(\beta=.699)$

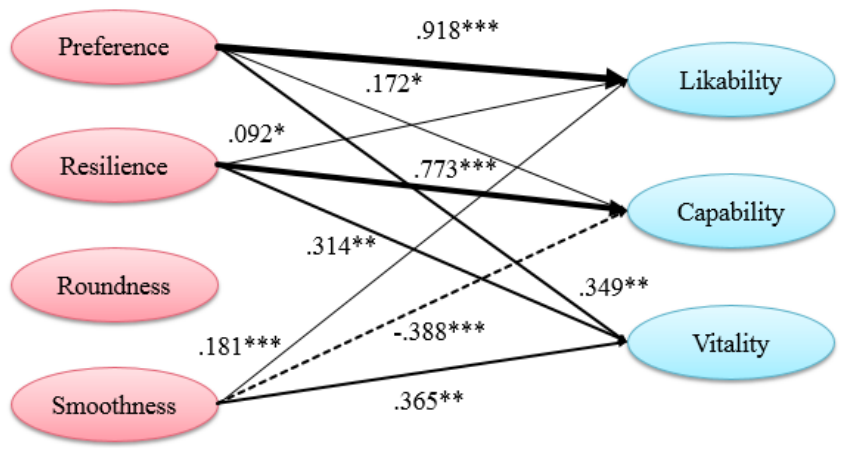

Figure 3. Path diagram with path coefficients representing the relationships between touch sensation factors and personality impression factors (in the Android condition). The insignificant paths were removed. ${ }^{*} \mathbf{p}<0.05, * * p<0.01, * * * p<0.001$ 


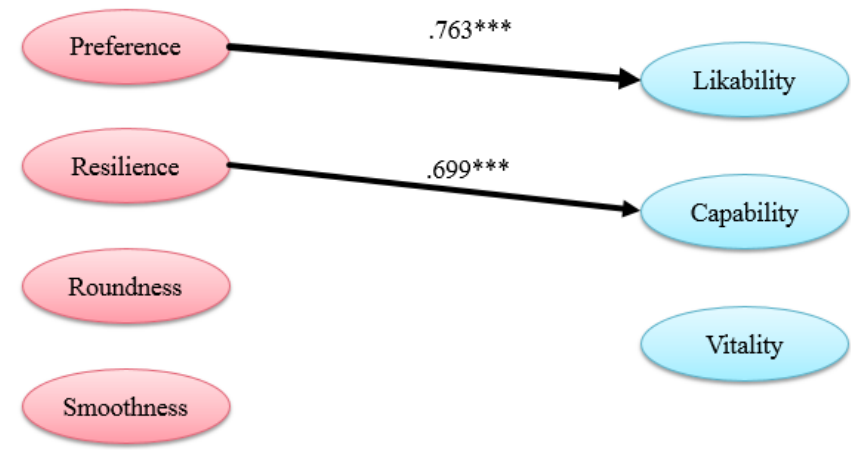

Figure 4. Path diagram with path coefficients representing the relationships between touch sensation factors and personality impression factors (in the Humanoid condition). The insignificant paths were removed. $* * * \mathbf{p}<0.001$

\section{DISCUSSION}

\section{Factor analysis}

Most of the found factors in touch sensation and personality impression were consistent with the factors found in other studies although the number of the experimental conditions of the forearm and appearance were limited in this study. Shirado and Maeno [21] reported that Rough, Cold, Moist, and Hard touch sensation factors were found by a factor analysis with a variety of materials such as paper, rubber, metal, wood, leather, styrene foam, sponge and plastic. The Rough and Hard touch sensation factors were equivalent to the Smoothness and Resilience touch sensation factors found in this study, respectively. The Cold and Moist touch sensation factors, which were not found in this study, would be found to analyze causal relationships including these two factors if we prepared other forearm conditions, such as with different temperature and moistness, and the adjectives related to these factors. On the other hand, the personality impression factors found in this study were consistent to the three factors, which were Evaluation, Potency, and Activity factors, known as typically-found factors in SD method [18]. This means that the adjective list on personality impression was enough to evaluate the robot comprehensively to some extent. Adding adjectives related to Familiarity and Enjoyment would enable us to further investigate the causal relationships in more detail because the Evaluation factor was found as two factors, which were Familiarity and Enjoyment, in Kanda et al.'s study on evaluation of social humanoid robot [9].

\section{Path analysis}

We found common significant causal relationships between touch sensations and personality impressions, which exist in both conditions. The first causal relationship was between the Preference touch sensation and the Likability personality impression. This relationship had high positive regression coefficients $(\beta=.918$ and .763 in the Android and Humanoid conditions, respectively). In other words, the Likability personality impression was improved by the Preference touch sensation in both conditions. The second causal relationship was between the Resilience touch sensation and the Capability personal impression. This relationship also had high positive regression coefficients $(\beta=.773$ and .669 in the Android and Humanoid conditions, respectively); the Capability personality impression was improved by the Resilience touch sensation in both conditions. This result suggests that these two causal relationships are more universal than the other significant causal relationships found only in the Android condition. Robot designers should be mindful of the differences in universality of the causal relationships between touch sensations and personality impressions: Universal causal relationships would be used to determine appropriate touch sensations for any other humanoid robots that give intended personality impressions, whereas non-universal ones should be investigated for each robot. Further analyses are necessary to investigate the degrees of the universalities for other types of robots including adult-type and animal robots with different movements and voices.

Several causal relationships that were significant in the Android condition were insignificant in the Humanoid condition. One possible reason of this is the weirdness of the facial appearance in the Humanoid condition, where the internal mechanisms of the face were exposed. This is because stronger attention to the weird facial appearance is considered to disturb the participants' evaluations of the robot. Collecting evaluation scores of the appearance of the robot is one of the future issues to conclude this.

\section{Conclusion}

Two common causal relationships between touch sensations and personality impressions were found in two conditions in which a child android robot had different appearances (Android condition with facial skin and Humanoid condition without facial skin). The Preference touch sensation improves the Likability personality impression and the Resilience touch sensation improves the Capability personality impression. On the other hand, other significant causal relationships were found only in the Android condition. This result suggests that two types of causal relationships exist between touch sensations and personality impressions: appearance-dependent relationships and appearance-independent relationships. Robot designers should mindful of the differences in universality of the causal relationships.

\section{ACKNOWLEDGMENTS}

This research is supported by PRESTO, JST (Grant Number JPMJPR1652) and by the Center of Innovation Program from MEXT and JST.

\section{REFERENCES}

1. Bartneck C, Kanda T, Ishiguro H and Hagita. 2007. Is the uncanny valley an uncanny cliff? In Proceedings of the 16th IEEE International Symposium on Robot and Human Interactive Communication: 368-373.

2. Castro-Gonzlez A, Admoni H and Scassellati B. 2016. Effects of form and motion on judgments of social robots' animacy, likability, trustworthiness and 
unpleasantness. International Journal of Human Computer Studies 90: 27-38.

3. Endo K, Endo N, Zecca M, Kusano T, MIzoguchi Y Ito $\mathrm{K}$ and Takanishi A. 2008. Design and development of the Waseda anthropomorphic Soft Hand WSH-1Evaluation of the interaction with young and elderly people- (in Japanese) . The Proceedings of the 26th Annual Conference of the Robotics Society of Japan AC1E2-03

4. Endo N, Iida F, Endo K, Mizoguchi Y, Zecca M and Takanishi A. 2010. Development of the anthropomorphic soft robotic hand WSH-1R. In Proceedings of the First IFToMM Asian Conference on Mechanism and Machine Science. p. 250162.

5. Iida F, Endo K, Mizoguchi Y, Endo N, Zecca M, Ito K and Takanishi A. 2009. Design and development of the anthropomorphic soft robot hand-Evaluation of the Effects of the Shape of the Artificial Hand- (in Japanese). The Proceedings of the 27th Annual Conference of the Robotics Society of Japan

6. Inoue $\mathrm{M}$ and Kobayashi T.1985. The Research domain and scale constriction of adjective-pairs in a semantic differential method in Japan (in Japanese). The Japanese Journal of Educational Psychology 33(3): 253-260.

7. Ishihara H and Asada M. 2015. Design of 22-DOF pneumatically actuated upper body for child android 'Affetto'. Advanced Robotics 29(18): 1151-1163.

8. Kamide H, Kawabe K, Shigemi S and Arai T. 2015. Anshin as a concept of subjective well-being between humans and robots in Japan. Advanced Robotics 29(24): 1624-1636.

9. Kanda T, Ishiguro H. and Ishida T. 2001. Psychological analysis on human-robot interaction. In Robotics and Automation, Proceedings ICRA. IEEE International Conference on Vol. 4: 4166-4173

10. Kanda T, Miyashita T, Osada T, Haikawa Y and Ishiguro H. 2008. Analysis of humanoid appearances in human-robot interaction. IEEE Transactions on Robotics 24(3): 725-735.

11. Kozima H, Michalowski MP and Nakagawa C. 2008. Keepon: A playful robot for research, therapy, and entertainment. International Journal of Social Robotics. 1(1): 3-18.

12. Kojima T, Endo K, Endo N, Iida F, Hashimoto K and Takanishi A. 2010. Design and development of the anthropomorphic soft robot hand-Evaluation of the Effect of the grip with grip strength control function (Translated from Japanese)- (in Japanese) The Proceedings of the 28th Annual Conference of the Robotics Society of Japan AC1O2-2
13. Lee J. K, Toscano R. L, Stiehl W. D and Breazeal C. 2008. The design of a semi-autonomous robot avatar for family communication and education. In Robot and Human Interactive Communication: 166-173.

14. Macdorman K. F. 2006. Subjective ratings of robot video clips for human likeness, familiarity, and eeriness: An exploration of the uncanny valley. In: Proceedings of the ICCS/CogSci-2006 Long Symposium: Toward Social Mechanisms of Android Science

15. Mori M. 2012. The uncanny valley. In K. F MacDorman and N. Kageki (Trans.), IEEE Robotics and Automation, 19(2): 98-100 (Original work published in 1970).

16. Mori Y, Saito Y and Kamide H. 2012. Evaluation of impression for hug dolls. Journal of Japan Society of Kansei Engineering 11(1): 9-15.

17. Niculescu A, Van Dijk B, Nijholt A and See S. L. 2011. The influence of voice pitch on the evaluation of a social robot receptionist. In Proceeding of the User Science and Engineering (i-USEr), 2011 International Conference on: 18-23.

18. Osgood C. E. 1952. The nature and measurement of meaning. Psychological Bulletin 49(3): 197-237

19. Piwek L, McKay L. S and Pollick F. E. 2014. Empirical evaluation of the uncanny valley hypothesis fails to confirm the predicted effect of motion. Cognition, 130(3), 271-277.

20. Shibata T and Wada K. 2011. Robot therapy: A new approach for mental healthcare of the elderly - a minireview. Gerontology 57(4): 378-86.

21. Shirado, H and Maeno, T. 2005. Modeling of human texture perception for tactile displays and sensors. In Eurohaptics Conference and Symposium on Haptic Interfaces for Virtual Environment and Teleoperator Systems. World Haptics. First Joint:: 629-630

22. Shirado H, Nonomura Y and Maeno T. 2007. Development of artificial skin having human skin-like texture. Transactions of the Japan Society of Mechanical Engineers Series C. 73(726): 541-546.

23. The R Foundation. The R project for statistical computing. 2016; 8/1(January):1-3. Available from: https://www.r-project.org/

24. Yamashita Y, Ishihara H, Ikeda T and Asada, M. 2016, November. Path analysis for the halo effect of touch sensations of robots on their personality impressions. In Proceedings of the International Conference on Social Robotics: 502-512 